\title{
A brief information-motivation-behavioral skills intervention to promote human papillomavirus vaccination among college-aged women
}

This article was published in the following Dove Press journal:

Psychology Research and Behavior Management

17 October 2016

Number of times this article has been viewed

\author{
Giselle K Perez' \\ Dean G Cruess ${ }^{2}$ \\ Nicole M Strauss ${ }^{3}$ \\ 'Department of Psychiatry, \\ Massachusetts General Hospital, \\ Boston, MA, ${ }^{2}$ Department of \\ Psychology, University of Connecticut, \\ Storrs, CT, ${ }^{3}$ Mongan Institute for \\ Health Policy, Massachusetts General \\ Hospital, Boston, MA, USA
}

Background: Human papillomavirus (HPV) is prevalent among college-aged women. Although HPV vaccines decrease women's risk for cervical cancer, the vaccination rates remain inadequate. Objective: This study explored the utility of an information-motivation-behavioral skills (IMB) intervention in promoting HPV vaccination knowledge, motivation, and intentions among college-aged women.

Methods: In Spring/Fall 2012, 62 participants were randomly assigned to a single-session intervention or attention control and were assessed baseline, post-intervention, and at 1 month.

Results: The participants demonstrated adequate baseline vaccine knowledge, low HPV/cancer knowledge, and ambivalence about the vaccination. Post-intervention, the IMB arm demonstrated increased HPV/cancer and vaccination knowledge, motivation, and intentions. There were no group differences in vaccination at 1 month; however, the odds of wanting to get vaccinated increased sevenfold in the IMB arm.

Conclusion: These results provide preliminary support for an IMB-based intervention in increasing vaccination knowledge, motivation, and intentions among at-risk women. Future research examining the efficacy of longer trials with larger, diverse populations is warranted.

Keywords: human papillomavirus, HPV, vaccination, cervical cancer, Gardasil, IMB

\section{Introduction}

Cervical cancer (CC) is predominantly a virally mediated disease with $99.7 \%$ of all cases having confirmed human papillomavirus (HPV) DNA. ${ }^{1}$ Considered as one of the most common sexually transmitted diseases, as many as $50 \%$ of sexually active individuals are infected with HPV in their lifetime. ${ }^{2}$ HPV infections are most prevalent in young adults, as sexual risk behaviors are greatest in this age group. ${ }^{3}$ Sexually active young women, in particular, carry the highest risk of infection, with studies documenting rates as high as $68 \%-71 \%$. ${ }^{4,5}$

HPV vaccines, such as the HPV quadrivalent known as Gardasil, provide women with a valuable tool to decrease their overall risk for $\mathrm{CC}$, as they protect against the strains (HPV 16 and 18) most strongly associated with the majority of HPV-related cancer cases. Accordingly, the HPV vaccine is recommended for girls aged 11-12 years, with catch-up vaccination for girls aged 13-26 years. ${ }^{6}$ Despite these guidelines, HPV vaccine uptake remains incomplete, and inadequate inoculation rates persist. ${ }^{7}$ In particular, women aged 18-26 years are less likely to initiate and complete the three-dose series than those aged 13-17 years. ${ }^{8}$ This represents an unmet need for strategies to improve HPV immunization, particularly among college-aged women.
Correspondence: Giselle K Perez Mongan Institute of Health Policy, Massachusetts General Hospital, 50 Staniford Street, Ninth Floor, Boston, MA 021 I4, USA

Tel + I 9782106520

Email gperez@mgh.harvard.edu 
Given the burden of HPV infections in young women and the clear link between HPV and CC, there is an emergent demand for the development and testing of evidence-based programs that directly support vaccination uptake. The President's Cancer Panel ${ }^{7}$ identified the need for increased vaccination as a "Public Health Priority." Likewise, a recent statement put forth by the American Society of Clinical Oncology emphasized the import of increasing HPV vaccination uptake. ${ }^{9}$ Despite these recommendations, there is scant research investigating the use of theory-based behavioral interventions to help stimulate Gardasil use among high-risk populations, such as young adult women.

The present study uses the information-motivationbehavioral skills (IMB) model as a theoretical framework to understand and elicit health behavior change. ${ }^{10}$ The IMB model proposes that an individual needs information about a target behavior, personal and social motivation to engage in the behavior, and behavioral skills to correctly perform the behavior in order to fully adopt a health behavior. ${ }^{10}$ Interventions founded on the IMB model address each of these integral components. The purpose of this study was to employ the IMB model to design, deliver, and explore the preliminary utility of a single-session intervention in improving Gardasil uptake among young women attending college in a rural setting.

\section{Methods}

\section{Participants and procedure}

The eligible participants consisted of a convenience sample of women, aged 18-26 years, enrolled in an Introductory Psychology course at a major state university in 2012 . Students who reported, through a university-wide screener, having received all three doses of the HPV vaccine were excluded from the study. Women interested in participating self-enrolled for a study session online via the university subject pool. Study sessions scheduled on the pool calendar corresponded to either an active intervention (IMB) or an attention control group; however, participants remained blinded to study condition to preserve random group assignment. The University of Connecticut's institutional review board approved the study procedures. This study was registered in ClinicalTrials.gov (NCT 02464358). All participants were individually consented by the study investigator prior to participation in all study activities.

\section{Treatment groups IMB group}

Federal law requires that a Vaccination Information Statement (VIS) be given to all individuals prior to receipt of any vaccine, including the HPV vaccine. ${ }^{11}$ The VIS is readily available online and in student health centers, and it provides a brief overview of HPV, HPV-related cancers, and the HPV vaccine. Both the IMB and attention control groups received this VIS prior to proceeding with study activities. In addition, the IMB group received: 1) supplementary educational content related to HPV and the HPV vaccine that addressed specific knowledge gaps identified among young adult women ${ }^{7,12}$; 2) motivational content, delivered using motivational interviewing (MI) techniques, ${ }^{13}$ to help women identify the benefits and barriers to vaccination; and 3) skills-building content, which included a review of, a) ways to access the HPV vaccine (eg, vaccine locations); b) methods for paying for the vaccine, including information related to insurance coverage and costs; c) reminder tools to ensure completion of the three-dose series; and d) approaches to communicating vaccine interest and concerns with parents and providers. The information was delivered in a small-group format within a university classroom to destigmatize HPV infection and to normalize HPV vaccination. Moreover, in accordance with the MI spirit, the entire intervention was delivered utilizing an air of acceptance, respect, and collaboration. Throughout group discussion, the facilitator incorporated several core MI skills, including asking open-ended questions, using reflective listening, and providing summaries, to highlight instances of ambivalence and to elicit self-change talk. In efforts to further tailor the intervention to meet the needs of participants, the group facilitator guided participants in problem-solving obstacles identified during group discussion. The session ended with asking students to consider what it would take to get vaccinated against HPV.

\section{Attention control group}

To maintain equivalence between study conditions, the attention control was also carried out in a small-group setting. Following receipt of the VIS, the participants watched a set of short video clips that covered broad topics related to women's health, including what happens during a sexual health check, ways to improve access to health services, and methods of contraception. Subsequently, the participants engaged in a brief group discussion, during which time the group facilitator addressed specific questions by directing the participants to particular sections of the videos or to websites readily available to the public.

\section{Measures}

The participants completed a questionnaire battery before (baseline: T1) and after (post-test: T2) participating in the 
IMB or attention control group as well as $\sim 4$ weeks later (T3). The study questionnaire assessed participants on a number of sociodemographic and personal health factors, including personal and familial cancer history, sexual health practices, and general health behaviors. In efforts to remain consistent with measures used in previous IMB studies, ${ }^{13-16}$ scales that tapped into each of the model's three dimensions were created by selecting and adapting questions used in other HPV and CC studies.

\section{Information}

Two scales were used to capture knowledge of HPV and the HPV vaccine. HPV vaccine knowledge was assessed with a nine-item scale consisting of true or false items. ${ }^{14}$ The items were summed to arrive at a total scale score (range 0-9). Knowledge of HPV and CC was measured with 15 items (five factual multiple-choice questions and ten true or false questions) taken from a 40-item self-report battery that measures knowledge, risk factors, perceived risk, and seriousness of HPV and CC. ${ }^{17}$ The number of correct responses was summed to obtain a total scale score for HPV/CC facts (range 0-15). These two scales were combined to create a total knowledge composite score.

\section{Motivation}

Consistent with the IMB model, motivation to get vaccinated was assessed with five scales that measured different facets of motivation: perceived motivation, attitudes toward vaccination, perceived social norms to getting vaccinated, behavioral intentions, and perceived risk for HPV/CC. Modeled after Kalichman et al, ${ }^{16}$ perceived motivation was assessed with a single item that asked participants to rank how motivated they would be from 1 (not at all) to 4 (very strong) to get the vaccine. Vaccination intentions were assessed through a series of six questions that asked participants about their intentions to gather more information about the vaccine, discuss the HPV vaccine with their friends, and get the HPV vaccine within the next year and/or within the next 3 years ("very unlikely" to "very likely" on a 6-point Likert scale). A seventh question specifically asked participants about their commitment to get vaccinated in the next year, and the response options included "undecided", "I do not want to get the vaccine", and "I want to get the vaccine". For the purposes of analyses, these responses were reordered to reflect contemplative movement, with "I do not want to get the vaccine" categorized as 0 , "undecided" as 1 , and "I want to get the vaccine" as 2 . This scale was modeled after a study aimed to reduce AIDS risk behavior in a college student population. ${ }^{18}$ The seven items were summed to arrive at a final score (range 6-39; $\alpha=0.89$ ). Attitudes toward vaccination were measured with a modified version of a scale used by Fisher et al. ${ }^{19}$ The participants were asked to rate their agreement with seven behaviors related to getting vaccinated (eg, getting more information about HPV vaccine, getting the HPV vaccine in the next month, discussing the vaccine with friends) on three 5-point Likert scales (eg, good-bad, nice-awful, pleasant-unpleasant). These items were summed to create a final measure of attitudes toward vaccination (range 21-105; $\alpha=0.95$ ), with higher scores indicating more negative attitudes. The perceived social norms were measured with another scale adapted from Fisher et al. ${ }^{19}$ The participants were asked to rate their level of agreement with seven behaviors associated with getting vaccinated (5-point Likert scale, very untrue to very true). The scale items were summed to arrive at a final measure of perceived social norms (range 7-35; $\alpha=0.91$ ). Perceived $\mathrm{CC}$ risk was determined by asking the participants to rate their level of agreement with nine statements (eg, "I believe that I am at risk for CC") on a 5-point Likert scale (strongly disagree to strongly agree). The nine items were summed to arrive at a total score (range $9-45 ; \alpha=0.76) .{ }^{17}$

\section{Behavioral skills}

Women's confidence in their ability to get vaccinated was assessed with two scales adapted from versions used by Fisher et al. ${ }^{18}$ Perceived efficacy was assessed by asking participants to rate how effectively they would be able to carry out nine behaviors associated with getting vaccinated (eg, how effectively can you get vaccinated against HPV, find the time to go to your provider for three visits to get vaccinated, afford to get vaccinated, etc) using a 5-point Likert scale ("very ineffectively" to "completely effectively"). The scale was averaged to arrive at a final scale score (range $1-5 ; \alpha=0.86$ ). Perceived difficulty was assessed by asking participants to rate how difficult they believe it would be to carry out the same nine behaviors associated with getting vaccinated (5-point Likert scale, "very hard to do" to "very easy to do"). The scale items were averaged to create a final score (range $1-5 ; \alpha=0.83$ ).

Vaccine uptake was measured at 4 weeks with a series of six yes/no questions related to the performance of several key vaccine-seeking behaviors, including taking steps to learn more about the vaccine; accessing the HPV vaccine; discussing the vaccine with family, friends, and providers; scheduling an appointment to get vaccinated; and initiating or completing the series. All items were summed, with higher values indicative of greater behavior change. 


\section{Analysis}

SPSS Version 20.0 (IBM Corporation, Armonk, NY, USA) was used to conduct all statistical analyses for this study. Prior to data analyses, all the data were screened for missing information and for violations of normality. Although some of the data were not consistent with a normal distribution, transformation of the scores did not influence study outcomes. Furthermore, transformation techniques are no longer generally recommended, especially when the tests used are predominantly robust by design. ${ }^{18}$ Accordingly, the analyses were conducted on original data.

Descriptive statistics, including frequencies, mean values, and ranges, were performed to describe the sample. Knowledge, motivation, and behavioral skills were assessed at baseline (T1), post-intervention (T2) and at 1 month (T3); behavior change was only assessed at 1 month. Group equivalence on relevant demographic factors and baseline IMB outcome variables was established using chi-square test/Fisher's exact tests for categorical variables and two sample $t$-tests for continuous variables. Baseline differences were discovered for age alone; as such, all subsequent multivariate analyses were adjusted for age. Furthermore, in efforts to control for potential confounders, we ran correlations between relevant sociodemographic and health history variables and each outcome variable; as recommended by Field, ${ }^{18}$ factors that were correlated with each outcome of interest at $P<0.05$ were included as covariates for related tests. Accordingly, vaccination status was included as a covariate across all tests, given its statistical and theoretical relationship with study outcomes. Pap screen history was included as a covariate for knowledge and behavioral skills due to its statistical and potential theoretical relationship. Though not statistically related, insurance status was included as a covariate for tests of group differences in behavioral skills, given its potential impact on perceived obstacles and the ability to get vaccinated. No other sociodemographic or health history factor was significantly correlated with our study outcomes.

A series of $2 \times 3$ repeated measures analysis of covariance (ANCOVA) were used to examine mean differences between groups on each of the composite factors of the IMB model: 1) knowledge; 2) motivation; and 3) behavioral skills. A Greenhouse-Geisser correction was applied when the assumption of sphericity was not met. For significant interactions, post hoc contrasts were used to identify linear or quadratic trends. A multivariate analysis of covariance (MANCOVA) was used to assess for group mean differences in the motivational construct, given the fact that the five scales were highly correlated. Doherty and Low suggest that MANCOVA is helpful for controlling type 1 error and for accounting for the correlation among these factors. ${ }^{20}$ Post hoc independent sample $t$-tests and/or pairwise $t$-tests were used to further explore significant group differences at each of the time points. When post hoc tests were conducted, the family-wise error rate was controlled for by adjusting the significance level to $P=0.03$ (ie, $0.05 / 2$ comparisons). All statistical tests were two tailed, and the significance level was set at $P<0.05$.

\section{Results}

\section{Characteristics of the sample}

A total of 70 women participated in the study (Table 1). Of them, 62 (89\%) completed the 1 month survey. Two thirds of the sample was Caucasian, and about $21 \%$ were Hispanic. The mean age was 19 years (range 18-26 years), and 75\% were in their freshman (48.4\%) or sophomore (25.8\%) year of college. The family income was high, with over half reporting an income $>\$ 75,000$.

Table I Sample characteristics

\begin{tabular}{|c|c|c|}
\hline Demographics & & \% Responders (n) \\
\hline \multirow[t]{2}{*}{ Age mean (range), years } & $|8-2|$ & $90.3(56)$ \\
\hline & $22-26$ & $9.7(6)$ \\
\hline \multirow[t]{5}{*}{ Race/ethnicity } & White/Caucasian & $66.1(4 I)$ \\
\hline & Black/African American & $8.1(5)$ \\
\hline & Asian & $21.0(13)$ \\
\hline & Hispanic & $21.0(13)$ \\
\hline & $\begin{array}{l}\text { Other (unidentified } \\
\text { multiracial) }\end{array}$ & $4.8(3)$ \\
\hline \multirow[t]{4}{*}{ Education } & Freshman & $48.4(30)$ \\
\hline & Sophomore & $25.8(16)$ \\
\hline & Junior & I6.I (I0) \\
\hline & Senior & $9.7(6)$ \\
\hline \multirow[t]{4}{*}{ Family income } & $<40,000$ & $23.3(14)$ \\
\hline & $40,000-74,999$ & $18.3(11)$ \\
\hline & $75,000-150,000$ & $41.7(25)$ \\
\hline & $>150,000$ & $16.7(10)$ \\
\hline \multirow[t]{2}{*}{ Health insurance } & No & $8.1(5)$ \\
\hline & Yes & $91.9(57)$ \\
\hline \multirow[t]{2}{*}{ Past Pap screen } & No & $72.6(45)$ \\
\hline & Yes & $27.4(17)$ \\
\hline \multirow{2}{*}{ History of genital warts } & No & $96.1(6 I)$ \\
\hline & Yes & $1.6(I)$ \\
\hline \multirow[t]{2}{*}{ History of STDs } & No & $96.8(60)$ \\
\hline & Yes & $3.2(2)$ \\
\hline \multirow[t]{2}{*}{ Ever had HPV test } & No & $91.9(57)$ \\
\hline & Yes & $8.1(5)$ \\
\hline Received at least one & No & $83.9(52)$ \\
\hline Gardasil dose & Yes & I6.I (10) \\
\hline
\end{tabular}

Abbreviations: STD, sexually transmitted disease; HPV, human papillomavirus. 


\section{Knowledge and awareness of HPV and Gardasil}

The majority of women had heard of HPV (87.1\%); however, few knew factual details about HPV (Tables S1 and S2). A sizable proportion was aware that HPV was sexually transmissible (75.2\%) and that it was associated with CC (71.0\%). In contrast, many women had heard of Gardasil (79\%) and were fairly knowledgeable about vaccine-related facts. Despite their awareness, $\sim 77.4 \%$ felt they had limited vaccine knowledge, and only $16.1 \%$ had received one dose of the vaccine. While few reported learning about the vaccine from their primary care physician (PCP; 27.4\%) or gynecologist (22.5\%), the majority (83.9\%) preferred to receive vaccine information from their PCP. Only half (56.5\%) had been recommended to get vaccinated from their PCP, and less than one-quarter were advised to get vaccinated by their gynecologists.

\section{Intervention effects on IMB}

\section{Information}

\section{Total knowledge scores}

A repeated measures ANCOVA was used to test for group differences in the mean knowledge scores over time after adjusting for age, Pap test experience, and vaccination status. There was a significant group by time effect for total HPV and Gardasil knowledge scores; $F(1.76,100)=4.69, P=0.02$, $\eta_{\mathrm{p}}{ }^{2}=0.076$. Simple effects analyses revealed that, compared to the attention control group, the participants in the IMB group demonstrated a significant improvement in knowledge scores from baseline to post-test $(P=0.05)$ and from baseline to 1 month $(P=0.005)$; however, there were no significant changes in knowledge scores from post-test to the 1 month follow-up for either group $(P=0.48)$. The results of post hoc analyses found no group differences in knowledge scores at baseline; however, the IMB group showed significantly higher knowledge scores at post-test $(M=16.43$ vs $M=14.86$; $P=0.03)$ and 1 month $(M=16.85$ vs $M=14.77 ; P<0.001$; Figure 1). Descriptive statistics for all main outcome measures by group are also presented in Table S3.

Separate ANCOVAs were conducted for the individual measures (ie, vaccine knowledge and knowledge of HPV and CC) used to arrive at the total knowledge score to examine if the intervention had a differential impact on HPV/HPV vaccine knowledge.

\section{Vaccine knowledge}

There was a significant group by time interaction for vaccine knowledge; $F(2,114)=3.88, P=0.02, \eta_{\mathrm{p}}{ }^{2}=0.064$. Simple effects analyses showed similar increases in vaccine knowledge from baseline to post-test for both groups, $P=0.99$; however, participants in the IMB group demonstrated continued improvement in vaccine knowledge from post-test to 1 month ( $M=5.42$ to $M=5.63 ; P=0.04$ ), whereas the control group demonstrated a decrease in vaccine knowledge $(M=5.71$ to $M=5.41$ ). Post hoc analyses found no significant group differences at any of the time points.

\section{HPV/CC knowledge}

A significant group by time interaction was found for HPV/ CC knowledge; $F(1.72,98.00)=4.33, P=0.02, \eta_{\mathrm{p}}{ }^{2}=0.071$. Simple effects analyses show that, relative to the control group, the IMB group had greater gains in HPV/CC knowledge from baseline to post-test $(P=0.03)$ and from baseline to the 1 month follow-up $(P=0.02)$; however, there were no significant changes in knowledge scores from post-test to 1 month for either group $(P=1.00)$. Post hoc analyses revealed greater vaccine knowledge scores at post-test ( $M=11.01$ vs $M=9.15 ; P=0.005)$ and at 1 month $(M=11.22$ vs $M=9.36$; $P<0.001)$ for the IMB group.

\section{Motivation}

A two-way, repeated measures MANCOVA was used to test for group differences on all five motivation constructs over time after adjusting for age and vaccination status. A significant group by time interaction was revealed; Pillai's trace $(10,47)=4.88, P<0.001, \eta_{\mathrm{p}}{ }^{2}=0.509$. Separate ANCOVAs were conducted for each of the motivational indices, and the findings are reported in the following (Figure 2).

\section{Self-reported vaccination motivation}

We found a significant group by time interaction for selfreported motivation, $F(2,112)=4.16, P=0.02, \eta_{\mathrm{p}}{ }^{2}=0.069$. Simple effects analyses revealed that, relative to the control group, the IMB group had a more pronounced increase in selfreported motivation from baseline to post-test $(P=0.008)$ and from baseline to 1 month $(P=0.04)$. Both groups demonstrated a similar decline in motivation from post-test to 1 month. Despite these trends, post hoc analyses found no significant group differences at any of the time points (Figure 2A).

\section{Vaccination intentions}

We found a significant group by time interaction for intentions to get vaccinated, $F(1.78,99.82)=7.40, P=0.002$, $\eta_{\mathrm{p}}{ }^{2}=0.117$. Specifically, the IMB group had greater increases in vaccination intentions from baseline to post-test $(P<0.001)$; 

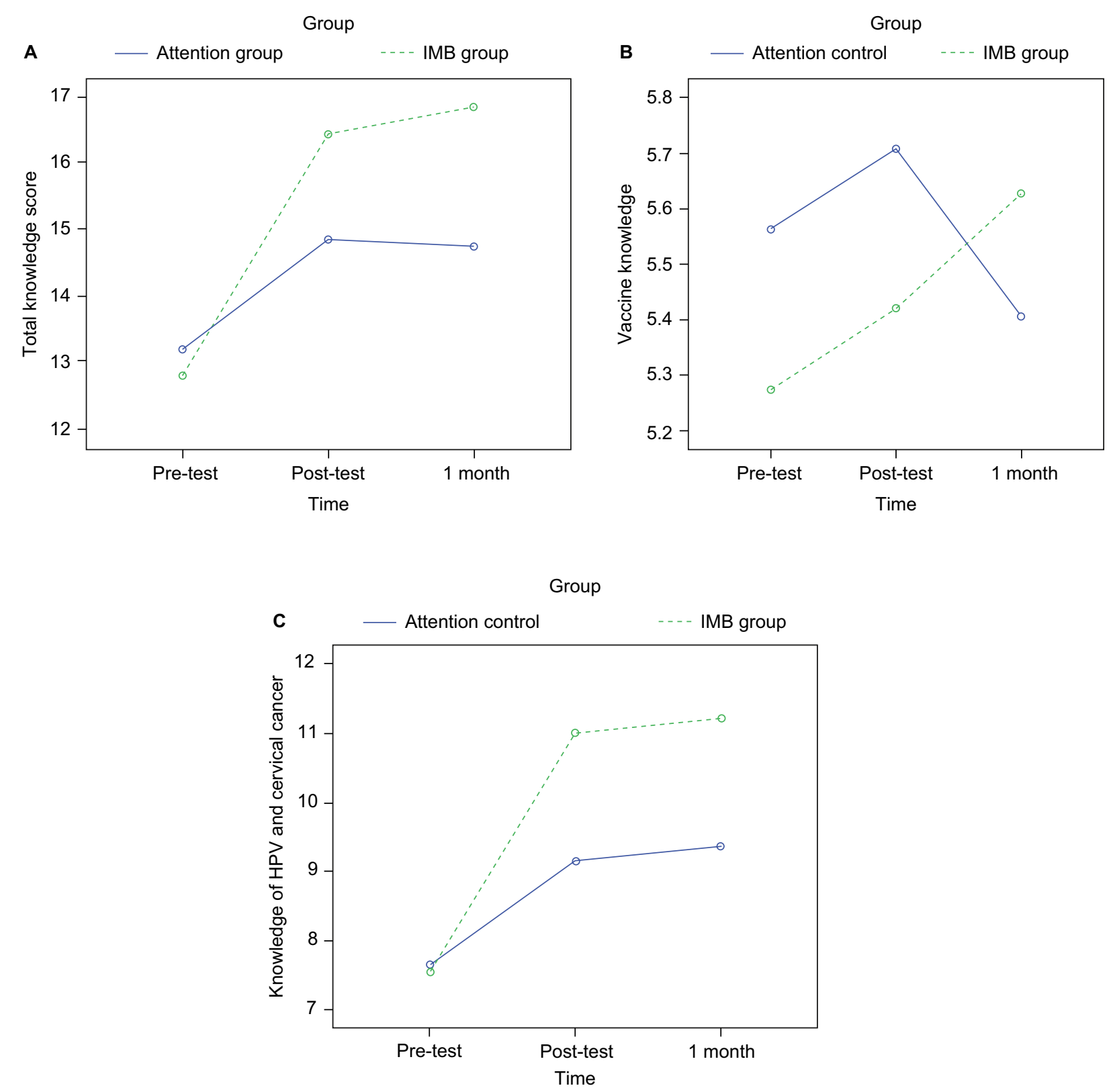

Figure I Estimated marginal mean values for (A) total knowledge scores, (B) vaccine knowledge, and (C) HPV/cervical cancer knowledge for condition across time. Abbreviations: HPV, human papillomavirus; IMB, information-motivation-behavioral skills.

however, they showed greater decreases in vaccination intentions from post-test to 1 month $(P=0.006)$. Vaccination intentions did not appear to change significantly from baseline to 1 month for either group $(P=0.47)$. Accordingly, post hoc analyses revealed no significant group differences in vaccination intentions at baseline and 1 month; however, the IMB group did endorse greater intentions to get vaccinated at post-test ( $M=29.12$ vs $M=24.34 ; P=0.03$; Figure 2B).

\section{Attitudes toward vaccination}

There was a significant group by time effect for attitudes toward getting vaccinated, $F(1.78,99.79)=7.11, P=0.002, \eta_{\mathrm{p}}{ }^{2}=0.113$. Specifically, while the control group did not demonstrate any significant changes in vaccination attitudes across time points, the IMB group showed significant improvements in attitudes toward vaccination from baseline to post-test $(P<0.001)$; however, these differences were not sustained. In fact, attitudes toward vaccination declined significantly from post-test to 1 month $(P=0.02)$. Accordingly, post hoc analyses revealed that, compared to the control group, the IMB group endorsed more positive attitudes toward HPV vaccination at post-test ( $M=36.35$ vs $M=51.41 ; P=0.007$; Figure 2C).

\section{Perceived social acceptability}

There were no significant group by time interactions for perceived social norms $(P=0.45$; Figure $2 \mathrm{D})$. 

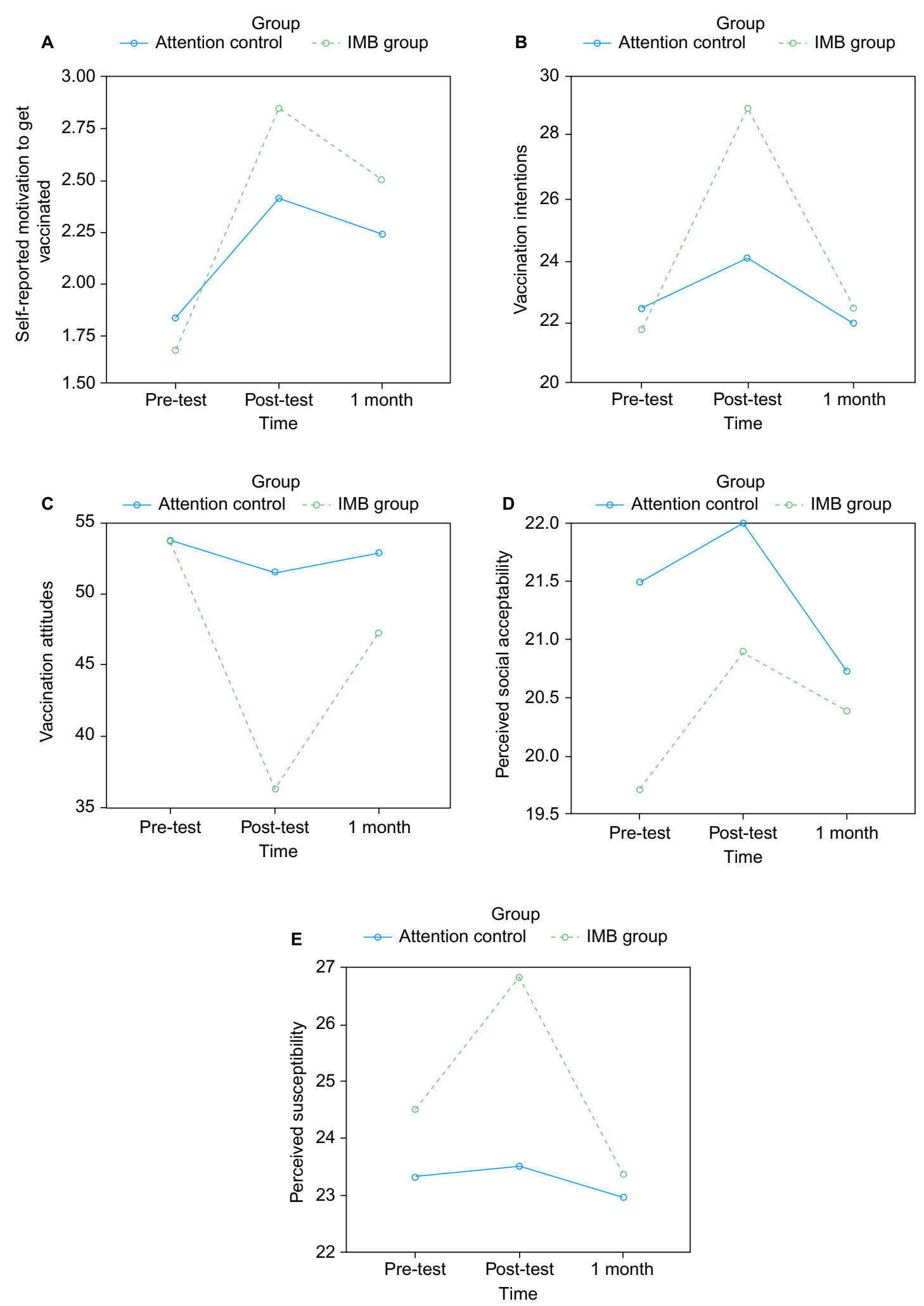

Figure 2 Estimated marginal mean values for (A) self-reported motivation, (B) vaccination intentions, (C) vaccination attitudes, (D) perceived social acceptability, and (E) perceived HPV/cervical cancer susceptibility for condition across time.

Abbreviations: HPV, human papillomavirus; IMB, information-motivation-behavioral skills. 


\section{Perceived susceptibility}

We found a significant group by time interaction for perceived susceptibility for HPV and $\mathrm{CC}, F(2,112)=3.13, P=0.05$, $\eta_{\mathrm{p}}{ }^{2}=0.053$. Specifically, while there were no significant changes in perceived risk for $\mathrm{HPV} / \mathrm{CC}$ for either group from baseline to post-test $(P=0.09)$ and from baseline to 1 month $(P=0.53)$, the IMB group demonstrated a greater decrease in perceived risk from post-test to 1 month $(P=0.01)$. Post hoc analyses revealed no significant group differences in perceived risk scores at baseline or 1 month; however, compared to the control group, the IMB group demonstrated higher HPV/CC risk perceptions at post-test ( $M=26.83$ vs $M=23.52 ; P=0.006$; Figure 2E).

\section{Behavioral skills}

There were no significant group by time effects for selfefficacy $(P=0.21)$ or perceived difficulty $(P=0.35)$ to get vaccinated (Figure 3 ).

\section{Behavioral intentions}

There were no significant differences in Gardasil uptake at 1 month $(P=0.89)$; however, women in the IMB group demonstrated greater intentions to get vaccinated, $\chi^{2}(2)=6.88$, $P=0.032$, and to engage in vaccine-seeking behaviors, $\chi^{2}(1)=6.61, P=0.01$. Based on the odds ratio, the odds of wanting to get vaccinated at the 1 month follow-up were 7.69 times higher if women participated in the IMB group compared to the attention control group. In addition, they were 6.90 times more likely to report intentions to engage in vaccinationseeking behaviors compared to women in the control group.

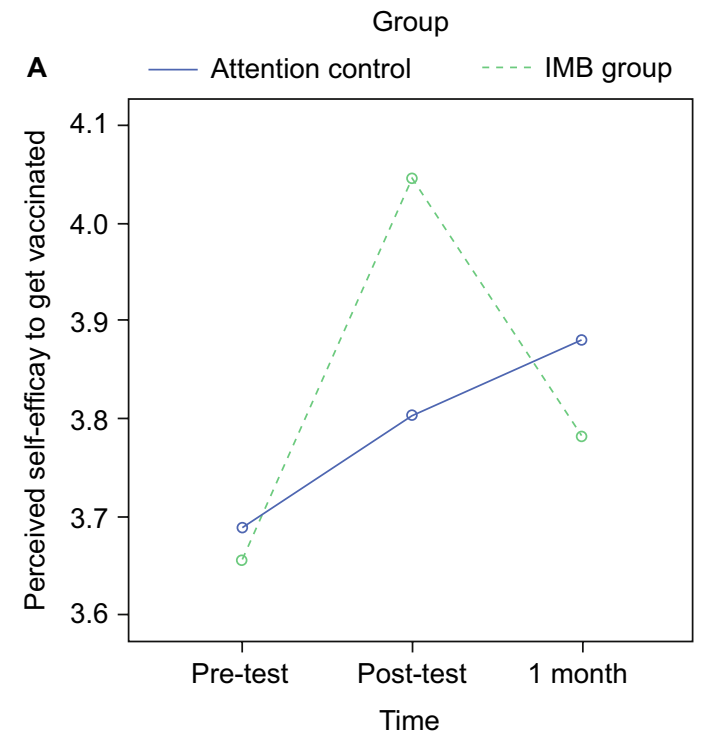

\section{Comment}

The purpose of this study was to examine the benefits of a theory-based, single-session, educational program in promoting Gardasil knowledge, motivation, and intentions to get vaccinated among college-aged women enrolled at a local university. Consistent with what has been reported in the literature, ${ }^{17,21}$ this sample of undergraduate women demonstrated high levels of vaccine knowledge in the context of having limited understanding of HPV and CC. As hypothesized, the IMB approach appeared to improve women's knowledge of Gardasil, HPV, and CC risk above and beyond that which can be attained through informational mediums (ie, HPV/ Gardasil VIS) that are readily available to the public via the Internet and in physician offices. Even more, the intervention appeared to contribute to sustained increases in vaccine knowledge despite the fact that both conditions received vaccine-specific information via the VIF. These findings suggest that participants in the IMB group may have derived additional benefits from the intervention. Specifically, it is possible that providing HPV vaccine information alone without highlighting the connection between HPV and CC may make it less likely that women will view the information as important and relevant. In fact, risk perception of HPV and $\mathrm{CC}$ was heightened for women in the IMB group immediately following the intervention. Accordingly, individuals who do not consider the information to be relevant may be less likely to process and review the information over time..$^{22} \mathrm{On}$ the other hand, the use of pamphlets or flyers alone may not be appropriate mediums for sharing information about HPV and the HPV vaccine. This may be partly due to the fact that

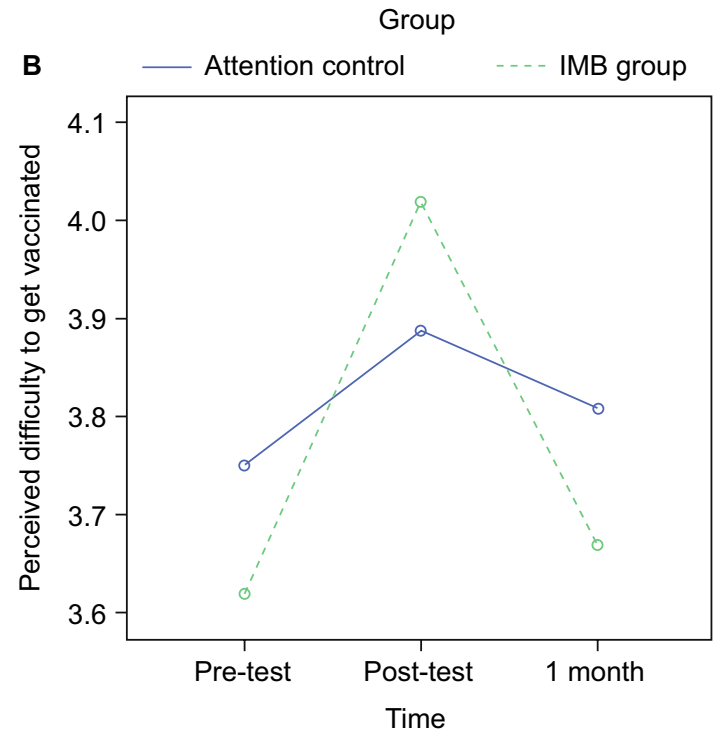

Figure 3 Estimated marginal mean values for (A) perceived self-efficacy and (B) perceived difficulty to get vaccinated for condition across time. Abbreviation: IMB, information-motivation-behavioral skills. 
few individuals may be interested in reading informational sheets, ${ }^{23}$ and many may lack the ability to fully understand the information. ${ }^{24}$ In fact, studies in the marketing literature indicate that individuals who are interested in the information presented are more likely to be more attentive and to demonstrate better information recall. ${ }^{22}$ Nonetheless, in light of the overall low-to-moderate levels of HPV knowledge reported for this high-risk population, ${ }^{17,21}$ these findings have important implications for cancer risk reduction, especially given the research linking knowledge of HPV and Gardasil to intentions to get vaccinated. ${ }^{25}$

Unlike the notable improvements we observed in knowledge scores, our theory that the intervention would enhance motivation to get vaccinated was only partly supported. Specifically, participants in the IMB condition demonstrated significant posttreatment changes in four of the five indices of motivation targeted by the intervention: self-reported motivation, intentions, attitudes toward vaccination, and perceived risk for HPV and CC. These findings corroborate existing studies that demonstrate similar motivational shifts following didactic strategies targeting Gardasil uptake. ${ }^{20,26-28}$ Interestingly, improvement in these four indices ceased postintervention; instead, we noted decreases in motivation scores over the 1 month follow-up. Although unexpected, it is likely that more than one intervention session is necessary to bring about enduring shifts in motivation. Nonetheless, these findings are important as the IMB model stresses the importance of motivation in driving behavior change. ${ }^{29}$

Contrary to our hypothesis, the intervention was no more effective than an attention control in engendering changes in social acceptability of Gardasil. In fact, perceived social norms toward vaccination increased uniformly across both conditions immediately following the manipulation. This outcome was surprising given the intervention's emphasis on destigmatizing HPV infection and vaccination behaviors by delivering risk and preventive information in a group setting; however, it is consistent with findings from other IMB-based interventions that also report similar challenges in eliciting changes in perceived norms for safe-sex behavior. ${ }^{19,30}$ Importantly, health education was delivered in a group format for both conditions, and this may have normalized all women's health topics regardless of subject matter. Although the control arm received limited information about HPV and Gardasil, it is plausible that general discussion of women's health themes in a peer setting is sufficient to modify perceived social norms for a myriad of health behaviors, including vaccine-seeking behaviors. The lack of observable change in social norms may also be due to limitations in the questionnaire battery. Specifically, it is possible that the questionnaire items used to assess for social norms tapped into an aspect of social acceptability that was not addressed by the intervention. ${ }^{19}$ Particularly, Krawczyk et al showed that parental approval and physician support are among the strongest indicators of vaccine behaviors. ${ }^{31}$ Qualitatively, many of our participants voiced an interest in pursuing Gardasil but were less likely to get vaccinated because their parents did not condone the vaccine and their physicians did not recommend it. This evidence is disconcerting, as physicians have demonstrated reluctance toward discussing the vaccine with their patients due to time constraints and perceived parental concerns. ${ }^{31,32}$ Accordingly, future research should include key individuals, such as parents, in these approaches in efforts to normalize HPV vaccination.

As hypothesized, women in the experimental arm reported significantly greater intentions to get vaccinated compared to women in the control arm. Moreover, these women also exhibited greater intentions to engage in vaccine-seeking behaviors, including obtaining more vaccine-specific information and discussing the vaccine with friends. These findings are noteworthy, as studies have identified a strong relationship between vaccination intentions and behaviors. ${ }^{28}$ Nonetheless, a number of barriers, including lack of physician and parental support, persist and likely contribute to the low vaccination rates widely reported. ${ }^{28}$ Accordingly, these findings underscore the importance of identifying and addressing these barriers in future interventions.

The IMB model contends that even well intentioned and knowledgeable individuals require a set of skills to enable them to engage in a health behavior. ${ }^{29}$ Behavioral skills are conceptualized as consisting of both objective and perceived (ie, self-efficacy) means of performing the behavior. At baseline, both groups did not appear to feel strongly about their ability to get vaccinated, nor did they consider the level of effort that may be involved in getting vaccinated. Following the manipulation, there were no significant group differences regarding perceived obstacles or self-efficacy to get vaccinated. Though surprising, this outcome is consistent with other studies. ${ }^{33,34}$ In fact, successful improvements in self-efficacy and perceived ease with engaging in target behaviors have been demonstrated in studies that had lengthier intervention approaches that were delivered over a span of 3-5 days. ${ }^{15,19}$ While there may be other factors that can explain this relationship, it is possible that these women did not have the opportunity to practice the skills they learned in session, thereby leading to limited changes in perceived effectiveness and feasibility relative to the control group. ${ }^{33}$ 
In regard to our final outcome, vaccine uptake, there were no group differences in Gardasil uptake at 1 month. This finding is likely due to the short follow-up period. It is possible that a longer follow-up is needed to allow for changes in vaccine uptake in this high-risk population. In fact, one study that explored the impact of an educational intervention on vaccine uptake in college students found no influence on vaccine uptake at 6 months despite the inclusion of a reminder letter. ${ }^{28}$ It is also possible that participants may have experienced other challenges and physical barriers to getting vaccinated, such as lack of time due to course load or attending school that is far from their gynecologist or PCP. Although participants can get the vaccine from local clinics, they may prefer to be treated by familiar physicians. ${ }^{35,36}$

\section{Limitations}

Despite the novelty of these findings, this study has some limitations. Because this study recruited from a convenience sample, study findings are limited in generalizability and may not reflect the opinions of women beyond this sample. Prior research has illustrated limited knowledge of HPV and underutilization of Gardasil among uninsured, low socioeconomic status, minority, and homosexual women ${ }^{37,38}$; thus, future studies should examine these attitudes among ethnic, socioeconomically, and sexually diverse groups. Moreover, future studies should be undertaken with men since vaccine recommendations extend to this group; vaccination of men can help reduce transmission of HPV infection. A further limitation of this study is the relatively small sample size; as such, caution should be taken when drawing conclusions from these findings. Future studies with a larger sample size will allow for exploration of additional factors (such as other health behaviors, including diet, exercise, sleep) that may influence vaccine uptake.

This study also has some methodological shortcomings. Specifically, despite good intentions, physical and academic obstacles may be realistic impediments to scheduling medical visits. Accordingly, a longitudinal study may be better equipped to ascertain these behavioral effects. ${ }^{28} \mathrm{~A}$ similar point is that this intervention was delivered over a brief, one-session visit. The majority of studies that employ the IMB model tend to deliver the IMB intervention over a span of several sessions. ${ }^{19,34}$ Nonetheless, while this can be considered a drawback, the development of brief programs to enhance $\mathrm{CC}$ preventive behaviors is warranted, given the need for practical, generalizable programs and the import of capturing the attention of this high-risk population. ${ }^{39}$ Last, future research should consider a comparison group that more closely resembles true standard of care, such as exposure to only the Gardasil VIF that is readily available in clinic offices. Efforts to maintain group equivalence in this study may have led to the inclusion of elements that generated improvements in the IMB indices for the control arm, thereby obfuscating group differences that would otherwise be larger. ${ }^{28}$

\section{Conclusion}

In spite of these limitations, this study is one of the first to explore the potential impact of a theory-based model in effecting changes in vaccine uptake among a high-risk population. These findings are consistent with current research that suggests that young women are generally aware of Gardasil, are less informed about HPV and CC, and are ambivalent about pursuing HPV vaccination. Although there were no discernible changes in vaccine uptake at 1 month, our preliminary findings suggest that interventions based on the IMB model may have the potential to influence young women's decisions to get vaccinated against HPV, a highly prevalent sexually transmitted disease in this population. As such, an IMB-based intervention may provide universities with a powerful strategy to motivate women to get vaccinated. Future studies may benefit from examining the efficacy of this program in a larger trial incorporating longer, followup periods to allow for adequate observation of behavioral change. Furthermore, future programs should also consider an optimal medium for delivering this intervention, such as through mobile health programs, to allow for easy integration and dissemination to students and their families during school-wide orientation periods, thereby providing universities with an inexpensive, brief, and effective program.

\section{Acknowledgment}

This project was supported through internal funds.

\section{Disclosure}

The authors report no conflicts of interest in this work.

\section{References}

1. Walboomers JM, Jacobs MV, Manos MM, et al. Human papillomavirus is a necessary cause of invasive cervical cancer worldwide. J Pathol. 1999;189(1):12-19.

2. Centers for Disease Control and Prevention. HPVInfection-Fact Sheet. CDC: Rockville, MD, USA; 2011.

3. Revzina NV, Diclemente RJ. Prevalence and incidence of human papillomavirus infection in women in the USA: a systematic review. Int $J$ STD AIDS. 2005;16(8):528-537.

4. Kahn JA, Rosenthal SL, Jin Y, Huang B, Namakydoust A, Zimet GD. Rates of human papillomavirus vaccination, attitudes about vaccination, and human papillomavirus prevalence in young women. Obstet Gynecol. 2008;111(5):1103-1110. 
5. Smith JS, Melendy A, Rana RK, Pimenta JM. Age-specific prevalence of infection with human papillomavirus in females: a global review. $J$ Adolesc Health. 2008;43(4 suppl):S5-S25.

6. (ACOG) AC of O and G. Committee opinion no. 641: human papillomavirus vaccination. Obstet Gynecol. 2015;126(3):e38-e43.

7. NCI [webpage on the Internet]. Accelerating HPV Vaccine Uptake: Urgency for Action to Prevent Cancer. 2014. http://deainfo.nci. nih.gov/advisory/pcp/annualReports/HPV/PDF/PCP_Annual_ Report_2012-2013.pdf. Accessed July 9, 2016.

8. Tan W, Viera AJ, Rowe-West B, Grimshaw A, Quinn B, Walter EB. The HPV vaccine: are dosing recommendations being followed? Vaccine. 2011;29(14):2548-2554.

9. Bailey HH, Chuang LT, duPont NC, et al. American Society of Clinical Oncology statement: human papillomavirus vaccination for cancer prevention. J Clin Oncol. 2016;34(15):1803-1812.

10. Fisher JD, Fisher WA. Changing AIDS-risk behavior. Psychol Bull. 1992;111(3):455-474.

11. Centers for Disease Control and Prevention C [webpage on the Internet]. Vaccine Information Statements (VIS). 2014. http://www.cdc.gov/vaccines/hcp/vis/about/facts-vis.html\#specific-vaccines. Accessed July 9, 2016.

12. Caskey R, Lindau ST, Alexander GC. Knowledge and early adoption of the HPV vaccine among girls and young women: results of a national survey. J Adolesc Health. 2009;45(5):453-462.

13. Carey MP, Braaten LS, Maisto SA, et al. Using information, motivational enhancement, and skills training to reduce the risk of HIV infection for low-income urban women: a second randomized clinical trial. Health Psychol. 2000;19(1):3-11.

14. Osborn CY, Amico KR, Cruz N, et al. A brief culturally tailored intervention for Puerto Ricans with type 2 diabetes. Health Educ Behav. 2010;37(6):849-862.

15. Fisher JD, Fisher WA, Misovich SJ, Kimble DL, Malloy TE. Changing AIDS risk behavior: effects of an intervention emphasizing AIDS risk reduction information, motivation, and behavioral skills in a college student population. Health Psychol. 1996;15(2):114-123.

16. Kalichman SC, Picciano JF, Roffman RA. Motivation to reduce HIV risk behaviors in the context of the Information, Motivation and Behavioral Skills (IMB) model of HIV prevention. J Health Psychol. 2008;13(5):680-689.

17. Gerend MA, Lee SC, Shepherd JE. Predictors of human papillomavirus vaccination acceptability among underserved women. Sex Transm Dis. 2007;34(7):468-471.

18. Field A. Discovering Statistics Using SPSS. 3rd ed. Sage Publications Ltd: London: 2009.

19. Fisher JD, Fisher WA, Bryan AD, Misovich SJ. Information-motivationbehavioral skills model-based HIV risk behavior change intervention for inner-city high school youth. Health Psychol. 2002;21(2):177-186.

20. Doherty K, Low KG. The effects of a web-based intervention on college students' knowledge of human papillomavirus and attitudes toward vaccination. Int J Sex Health. 2008;20(4):223-232.

21. Daley EM, Vamos CA, Buhi ER, et al. Influences on human papillomavirus vaccination status among female college students. J Womens Health (Larchmt). 2010;19(10):1885-1891.
22. Shavitt S, Vargas P, Lowrey P. Exploring the role of memory for selfselected ad experiences: are some advertising media better liked than others? Psychol Mark. 2004;21(12):1011-1032.

23. Warren K. HPV knowledge among female college students and the short term effectiveness of HPV education. Internet J Acad Physician Assist. 2009;7:1-8.

24. Waller J, Marlow LA, Wardle J. The association between knowledge of HPV and feelings of stigma, shame and anxiety. Sex Transm Infect. 2007;83(2):155-159.

25. Wong LP, Sam I. Ethnically diverse female university students' knowledge and attitudes toward human papillomavirus (HPV), HPV vaccination and cervical cancer - ClinicalKey. Eur J Obs Gynecol Reprod Biol. 2010;148:90-95.

26. Cox DS, Cox AD, Sturm L, Zimet G. Behavioral interventions to increase HPV vaccination acceptability among mothers of young girls. Health Psychol. 2010;29(1):29-39.

27. Kennedy A, Sapsis KF, Stokley S, Curtis CR, Gust D. Parental attitudes toward human papillomavirus vaccination: evaluation of an educational intervention, 2008. J Health Commun. 2011;16(3):300-313.

28. Patel DA, Zochowski M, Peterman S, Dempsey AF, Ernst S, Dalton VK. Human papillomavirus vaccine intent and uptake among female college students. J Am Coll Health. 2012;60(2):151-161.

29. Fisher J, Fisher W. Theoretical approaches to individual-level change in HIV risk behavior. CHIP Doc. 2000:3-55.

30. Miller WR, Rollnick S. Motivational Interviewing: Preparing People to Change Addictive Behavior. New York: Guilford Press; 1991.

31. Krawczyk AL, Perez S, Lau E, et al. Human papillomavirus vaccination intentions and uptake in college women. Health Psychol. 2012;31(5):685-693.

32. Quinn GP, Murphy D, Malo TL, Christie J, Vadaparampil ST. A national survey about human papillomavirus vaccination: what we didn't ask, but physicians wanted us to know. J Pediatr Adolesc Gynecol. 2012;25(4):254-258.

33. Jaworski BC, Carey MP. Effects of a brief, theory-based STDprevention program for female college students. J Adolesc Health. 2001;29(6):417-425.

34. O'Grady MA, Wilson K, Harman JJ. Preliminary findings from a brief, peer-led safer sex intervention for college students living in residence halls. J Prim Prev. 2009;30(6):716-731.

35. Hollander D. Provider recommendation linked to adolescent-females' HPV vaccination. Perspect Sex Reprod Health. 2012;44(3):208-209.

36. Rosenthal SL, Weiss TW, Zimet GD, Ma L, Good MB, Vichnin MD Predictors of HPV vaccine uptake among women aged 19-26: importance of a physician's recommendation. Vaccine. 2011;29(5):890-895.

37. Cook RL, Zhang J, Mullins J, et al. Factors associated with initiation and completion of human papillomavirus vaccine series among young women enrolled in Medicaid. J Adolesc Health. 2010;47(6):596-599.

38. McNair R, Power J, Carr S. Comparing knowledge and perceived risk related to the human papilloma virus among Australian women of diverse sexual orientations. Aust N ZJ Public Health. 2009;33(1):87-93.

39. Moore EW, Smith WE, Folsom AR. F.O.R.E.play: the utility of brief sexual health interventions among college students. J Am Coll Health. 2012;60(2):175-177. 


\section{Supplementary materials}

Table SI General knowledge of HPV and CC

\begin{tabular}{ll}
\hline Knowledge items & \% Correct (n) \\
\hline The virus associated with CC is transmitted by sex & $72.5(45)$ \\
CC and precancer cells are associated with HPV & $71.0(44)$ \\
CC can be diagnosed by Pap tests & $74.2(46)$ \\
CC prevention requires delayed sex, Pap tests, and & $74.2(46)$ \\
condom use & \\
HPV can cause genital warts & $40.3(25)$ \\
HPV can live in skin without causing growths or & $48.4(30)$ \\
changes & \\
Multiple sex partners increases risk for CC & $77.4(48)$ \\
Having genital warts increases risk for CC (false) & $9.7(6)$ \\
Having sex before age I8 increases risk for CC & $45.2(28)$ \\
Taking illegal drugs increases risk for CC (false) & $41.9(26)$ \\
Having any STD increases CC risk & $71.0(44)$ \\
Smoking cigarettes increases risk for CC & $12.9(8)$ \\
Poor diet or nutrition increases risk for CC (false) & $45.2(28)$ \\
Using tampons increases risk for CC (false) & $71.0(44)$ \\
Using oral contraceptives increases risk for CC & $4.8(3)$ \\
\hline
\end{tabular}

Abbreviations: HPV, human papillomavirus; CC, cervical cancer; STD, sexually transmitted disease.
Table S2 General knowledge of HPV vaccine

\begin{tabular}{ll}
\hline Knowledge items & \% Correct (n) \\
\hline $\begin{array}{l}\text { Girls/women who get the HPV vaccine need less } \\
\text { frequent pelvic examinations (false) }\end{array}$ & $91.9(57)$ \\
Girls/women who get the HPV vaccine do not have & $98.4(6 \mathrm{I})$ \\
to get Pap smears (false) & $96.8(60)$ \\
$\begin{array}{l}\text { The HPV vaccine protects against all sexually } \\
\text { transmitted infections (false) }\end{array}$ & $71.0(44)$ \\
$\begin{array}{l}\text { The HPV vaccine protects against CC (true) } \\
\text { Girls/women who get the HPV vaccine can worry }\end{array}$ & $83.9(52)$ \\
less about all STDs (false) & $100.0(62)$ \\
$\begin{array}{l}\text { Girls who get the HPV vaccine no longer need } \\
\text { condoms during sex (false) }\end{array}$ \\
\hline $\begin{array}{l}\text { Abbreviations: HPV, human papillomavirus; CC, cervical cancer; STD, sexually } \\
\text { transmitted disease. }\end{array}$
\end{tabular}

Table S3 Intervention effects on vaccination IMB

\begin{tabular}{|c|c|c|c|c|c|c|c|c|c|c|c|c|}
\hline \multirow[t]{4}{*}{ Measure } & \multicolumn{6}{|c|}{ IMB arm } & \multicolumn{6}{|c|}{ Attention control } \\
\hline & \multicolumn{2}{|l|}{$\overline{T I}$} & \multicolumn{2}{|l|}{ T2 } & \multicolumn{2}{|l|}{ T3 } & \multicolumn{2}{|l|}{ TI } & \multicolumn{2}{|l|}{ T2 } & \multicolumn{2}{|l|}{ T3 } \\
\hline & \multicolumn{2}{|c|}{ Baseline } & \multicolumn{2}{|c|}{$\overline{\text { Post-intervention }}$} & \multicolumn{2}{|c|}{ I month } & \multicolumn{2}{|c|}{ Baseline } & \multicolumn{2}{|c|}{$\overline{\text { Post-intervention }}$} & \multicolumn{2}{|c|}{ I month } \\
\hline & $M$ & SE & $M$ & SE & $M$ & SE & $M$ & SE & $M$ & SE & $M$ & SE \\
\hline \multicolumn{13}{|l|}{ Information } \\
\hline Total score & 12.82 & 0.58 & 16.43 & 0.48 & 16.85 & 0.34 & 13.22 & 0.58 & 14.86 & 0.48 & 14.77 & 0.34 \\
\hline Vaccine knowledge & 5.27 & 0.12 & 5.42 & 0.12 & 5.63 & 0.12 & 5.56 & 0.12 & 5.71 & 0.12 & 5.41 & 0.12 \\
\hline HPV/CC knowledge & 7.54 & 0.53 & 11.01 & 0.43 & 11.22 & 0.31 & 7.65 & 0.53 & 9.15 & 0.43 & 9.36 & 0.31 \\
\hline \multicolumn{13}{|l|}{ Motivation } \\
\hline Motivation & 1.67 & 0.14 & 2.85 & 0.19 & 2.53 & 0.19 & 1.86 & 0.14 & 2.44 & 0.19 & 2.25 & 0.18 \\
\hline Intentions & 21.81 & 1.34 & 29.12 & 1.42 & 22.42 & 1.37 & 22.60 & 1.29 & 24.34 & 1.37 & 21.93 & 1.32 \\
\hline Attitudes & 53.78 & 3.56 & 36.35 & 3.61 & 47.25 & 3.72 & 53.69 & 3.44 & 51.41 & 3.48 & 52.90 & 3.60 \\
\hline Social norms & $19.7 \mid$ & 1.03 & 20.90 & 1.11 & 20.39 & 1.26 & 21.50 & 0.99 & 22.00 & 1.07 & 20.73 & 1.22 \\
\hline Susceptibility & 24.51 & 0.94 & 26.83 & 0.98 & 23.38 & 1.04 & 23.33 & 0.90 & 23.52 & 0.95 & 22.97 & 1.01 \\
\hline \multicolumn{13}{|l|}{ Behavioral skills } \\
\hline Perceived efficacy & 3.66 & 0.14 & 4.05 & 0.13 & 3.78 & 0.14 & 3.69 & 0.14 & 3.80 & 0.13 & 3.88 & 0.14 \\
\hline Perceived difficulty & 3.62 & 0.13 & 4.02 & 0.13 & 3.67 & 0.15 & 3.75 & 0.13 & 3.89 & 0.13 & 3.81 & 0.15 \\
\hline
\end{tabular}

Abbreviations: IMB, information-motivation-behavioral skills; SE, standard error; HPV, human papillomavirus; CC, cervical cancer.

Psychology Research and Behavior Management

Dovepress

\section{Publish your work in this journal}

Psychology Research and Behavior Management is an international, peerreviewed, open access journal focusing on the science of psychology and its application in behavior management to develop improved outcomes in the clinical, educational, sports and business arenas. Specific topics covered in the journal include: Neuroscience, memory and decision making; Behavior modification and management; Clinical applications; Business and sports performance management; Social and developmental studies; Animal studies. The manuscript management system is completely online and includes a very quick and fair peer-review system, which is all easy to use. Visit http://www. dovepress.com/testimonials.php to read real quotes from published authors. 